\title{
How Often Excitation Precipitates Seizure? Relative Difference of Frequency and Significance in Different Types of Epilepsy
}

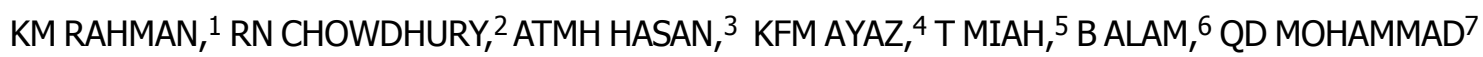

\begin{abstract}
:
Background: Epilepsy is a chronic neurological disorder, which presents with recurrent, unprovoked seizures. Seizure occurs spontaneously usually, but there may be association of various triggers. These triggers may act as seizure precipitating factors (SPFs).

Objective: To find out the frequency of excitation as a SPF and its relationship to different types of epileptic patients attending Neurology and Medicine outpatient department (OPD) of a tertiary care hospital in Bangladesh.

Methods: This was a cross sectional study carried out in specialized epilepsy weekly outdoor clinic of Neurology and Medicine outpatient department (OPD) of Dhaka Medical College Hospital from January 2008 to July 201 I. Epilepsy syndromes were broadly classified into four categories using the classification system of International League Against Epilepsy (ILAE) namely, generalized epilepsy (GE), localization related epilepsy (LRE), symptomatic and unclassified. Each patient was evaluated and classified by consultant neurologist. All participants were interviewed through a predesigned close ended questionnaire to identify frequency of excitation as a SPF.

Result: Among all the patients 7 I.5\% were male, with most common age group at the time of interview was I I-20 years $(36.8 \%)$. About $62 \%$ could identify at least one precipitator. Excitation (25.2\%) was the most common SPF reported by patients followed by emotional stress in 17.7\%. Excitation was found to be significantly associated with generalized epilepsy $(p<.000)$.
\end{abstract}

Conclusion: Most of the patients can identify their seizure precipitant. Excitation showed a strong association with generalized epilepsy.

Keyword: Generalized epilepsy (GE), Localization related epilepsy (LRE)

\section{Introduction:}

A seizure is any clinical event due to abnormal discharge of cerebral neurons. ${ }^{1}$ The threshold or susceptibility for seizure events varies among population which implies the influence

1. Assistant Professor of Neurology, Dhaka Medical College Hospital

2. Assistant Professor of Neurology, Dhaka Medical College Hospital

3. Medical Officer (Medicine OPD), Dhaka Medical College Hospital

4. Assistant Professor of Medicine, Dhaka Medical College Hospital

5. Assistant Professor of Medicine, Dhaka Medical College Hospital

6. Associate Professor of Neurology, Dhaka Medical College Hospital

7. Professor Head, Dept of Neurology, Dhaka Medical College Hospital

Correspondence : Dr. Rajib Nayan Chowdhury, Assistant Professor of Neurology, Dhaka Medical College Hospital. Email: rajibchow86@yahoo.com of various endogenous or exogenous factors. Patients with epilepsy experience recurrent, unprovoked seizures. Though most occur spontaneously, it is now well accepted that there is association of various triggers. The seizure precipitating factor (SPF) may be defined as any endogenous or exogenous factors that promote the occurrence of epileptic seizures. Some authors have defined these factors as "those circumstances that precede the onset of an epileptic attack and are considered by both patient and neurologist to be a possible explanation for why the seizure happened when it did, and not earlier or later. ${ }^{2}$ These include both seizure inducing factors (environmental or exogenous) and seizure triggering factors (chemical or physiological stimuli). ${ }^{3}$ Identification, analysis and distribution of these factors in various epilepsy and epilepsy syndromes will help in better understanding of patho-physiologic mechanism of seizure. Excitation in any form is thought to be a seizure precipitating factor. This study was designed to investigate frequency and association of excitation as a SPF in different types of epilepsy. 


\section{Material and methods:}

This cross sectional study was carried out in specialized epilepsy weekly outdoor clinic and Medicine outpatient department (OPD) of Dhaka Medical College Hospital from January 2008 to July 2011, which included 584 epilepsy patients. Epilepsy syndromes were broadly classified into four categories using the classification system of International League Against Epilepsy (ILAE) namely, generalized epilepsy (GE), localization related epilepsy (LRE), symptomatic and unclassified. Each patient was evaluated and classified by consultant neurologist. All participants with history of seizure were interviewed through a predesigned close ended questionnaire inquiring about factors that they believed to influence the occurrence of seizure events in own cases. Whereas, non-cooperative patients were excluded from study. Statistical analysis of their response was done with the help of SPSS version 16.0.

\section{Results:}

This study was carried out among 584 subjects, and out of all $71.5 \%$ was male. The most common age group at the time of interview was $11-20$ years (36.8\%), followed by $27.7 \%$ young adults who were at 21-30 years of age (Table-I). Only $29(2.5 \%)$ respondents were older than 60 years. When the patients were asked about the factors that they think to be responsible for seizure, about $62 \%$ could indentify at least one precipitator. Excitation $(25.2 \%)$ was the most

\section{Table-I}

Socio demographic profile of the patients $(N=584)$

\begin{tabular}{llcc}
\hline Parameter & $\mathrm{n}$ & $\%$ \\
\hline Age & & & \\
& 0-10yr & 114 & 19.5 \\
& 11-20yrs & 225 & 38.5 \\
& 21-30yrs & 161 & 27.5 \\
& 31-40yrs & 55 & 9.4 \\
& 41-50yrs & 15 & 2.6 \\
& $>60$ yrs & 14 & 2.5 \\
Sex & & & \\
& Male & 417 & 71.5 \\
& Female & 167 & 28.5 \\
\hline
\end{tabular}

Table-I Shows that among all participants commonest age group was 11-20 years with a frequency of $38.5 \%(n-225)$, and male patients were $71.5 \%(n-417)$. common SPF reported by patients followed by emotional stress in $17.7 \%$. Fever precipitated seizure in $16.4 \%$ of the subjects. A good number of them (15.5\%) noticed that whenever there was sleep deprivation, they had an attack of epilepsy. However, drug withdrawal, playing outside and head trauma were also common among the patients, about $11.7 \%, 11.3 \%$ and $8.4 \%$ respectively (Table-II). Apart from these television watching, sunshine/ flickering light, infection, hyperventilation was also reported by some. But none reported alcoholism or alcohol withdrawal, menstruation, auditory stimuli, touch, sexual intercourse, tiredness, pregnancy, drug, constipation, fasting, pain, startle or fright, electrolyte imbalance etc, as the cause of their problem. Subgroup analysis showed a significant difference in distribution of SPF among different epilepsy classes. Excitation was found to be a SPF in 224 patients of GE and 55 patients of LRE. Excitation showed a strong association in generalized epilepsy $(p$ value $=0.000)$ (Table-III).

Table-II

Common seizure precipitators in Epileptic patients $(N=584)$

\begin{tabular}{lcc}
\hline Parameter & $\mathrm{n}$ & $\%$ \\
\hline Excitation & 147 & 25.2 \\
Emotional stress & 104 & 17.7 \\
Fever & 96 & 16.4 \\
Sleep deprivation & 90 & 15.5 \\
Drug withdrawal & 68 & 11.7 \\
Playing & 66 & 11.3 \\
Head trauma & 52 & 8.9 \\
Watching television & 29 & 5.9 \\
Sunshine/ Flickering light & 22 & 4.5 \\
Physical exercise & 12 & 2 \\
Infection & 11 & 2 \\
Breath holding & 7 & 1.2 \\
Hyperventilation & 4 & 0.7 \\
Others & 25 & 4.4 \\
\hline
\end{tabular}

Table-II Shows common seizure precipitating conditions in epilepsy patients. Excitation is the most common condition. 
Table III

Association of excitation in different types of epilepsy

\begin{tabular}{llcccc}
\hline Parameter & \multicolumn{5}{c}{ Epilepsy class } \\
\cline { 2 - 5 } & $\mathbf{G}$ & LRE & Symptomatic & Unclassified & $p$ value \\
\hline Excitation & 112 & 27 & 8 & 0 & 0.00 \\
\hline
\end{tabular}

Table-III Shows distribution and association of excitation with different epilepsy types

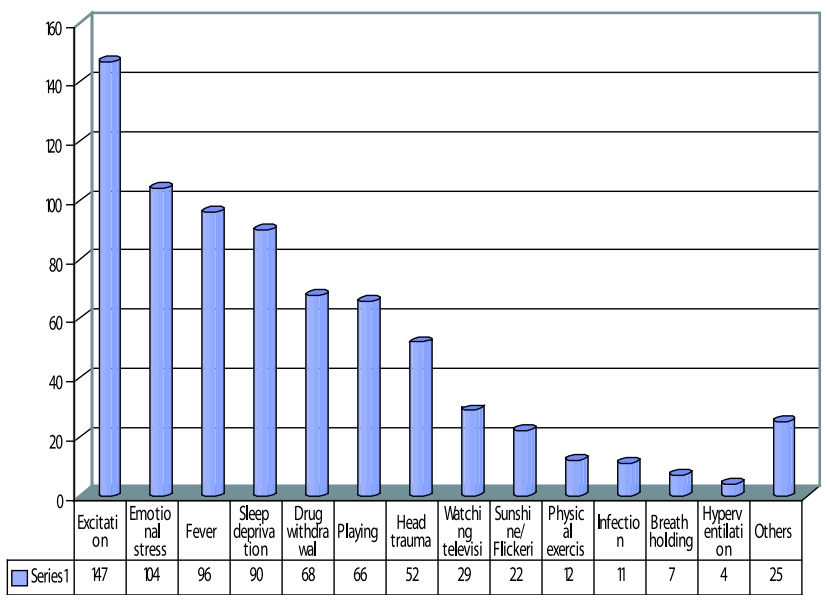

Fig.-1: Seizure precipitators

Figure-I Shows frequency of different siezure precipitating conditions in epilepsy patients (n-584).

\section{Discussion:}

SPFs have raised interest among the researchers. There are several potential causes of a seizure event which include genetic predisposition, underlying central nervous system lesion, one or more precipitating factors and or combination of all these factors that may decrease the seizure threshold in an individual. Sometimes it is difficult for the patient to identify or recall any factor that might have facilitated a seizure. Literatures regarding SPFs showed almost 40 factors that may actually potentiate a seizure in susceptible individual. ${ }^{3}$ Moreover one precipitator may potentiate other events in same individual eg emotional stress and excitation may lead to sleep deprivation and drug noncompliance. Here we tried to investigate about the self perception of SPF among the epilepsy patients. Similar to other reported studies, about $2 / 3^{\text {rd }}$ of the respondents in this study identified at least one precipitating factor which varies from $61-100 \%$ in different series. ${ }^{4,5}$ Although many series of study reported emotional stress as most common seizure precipitant, ${ }^{6,7}$ we found excitation instead as a precipitant in this sample. The difference might account for the variation in genetic and environmental factors in different part of the world. Emotional stress though not common as the other reports, still a good number of patients mentioned it.

Not surprisingly like other studies, some precipitants e.g. excitation, emotional stress, sleep deprivation clustered together in patients which suggests that they share similar pathophysiologic mechanism and act in similar fashion in different seizure types. ${ }^{8}$ But evidence is limited whether avoiding these factors actually reduce seizure frequency in epilepsy patients. This demands further multicentre studies to comment on this matter in future. Nevertheless the sleep deprivation appears to induce interictal epileptiform discharges. ${ }^{9}, 10$ Here excitation strongly correlated with GE ( $p$ value was significant). Although the finding corresponds with the common knowledge about precipitating factors in GE, the explanation behind this finding is largely unknown. ${ }^{11-}$ ${ }^{13}$ More than one third of respondents failed to identify any precipitant and the number was larger than any single seizure precipitant cited by them. This implies that seizure precipitants are modulators of seizure events rather than a trigger.

We had some limitations in this study. First of all, the availability of long term seizure diary was not possible in this setting. So we tried to minimize the recall bias from patients by presenting a list of factors. But on the other hand, a closed questionnaire tends to induce more false positive cases. Fortunately, the percentage of positive cases was within the range of other published studies. Secondly, the classification of epilepsy types and syndromes may not be uniform among the treating physicians. So we tried to stick to ILAE classification system. Therefore we believe that the distribution of excitation as SPFs is quite accurate and their influence on different epilepsy syndrome is well judged. Even though our study was based on self perception and did not have any control group, it showed that structured questionnaire is useful in stimulating patients thought and attitude to their problems.

\section{Conclusion:}

The relationship between seizure precipitants and seizure occurrence is a complex one. Very little is known about the actual mechanism by which these factors may influence the 
seizure threshold. Overall this study yielded important information. First of all, most patients can identify their seizure precipitant. Secondly, excitation is the most common SPF in this study. Finally, there is significant association of excitation with generalized epilepsy (GE).

\section{Conflict of Interest: None}

\section{References:}

1. Allen CMC, Lueck CJ, Dennis M. Neurologic disease. In: Colledge NR, Walker BR, Ralston SH, editors. Davidson's Principles and Practice of Medicine. $21^{\text {st }}$ edition. Elsevier Limited; 2010: pp-1172

2. Burdette DE, Feldman RG. Factors that can exacerbate seizures. In: Resor SR, Kutt H, editors. The medical treatment of epilepsy. New York: Marcel Dekker; 1992. p. 79-89

3. Aird RB. The importance of seizure-inducing factors in the control of refractory forms of epilepsy. Epilepsia 1983; 24: $567-83$

4. Murthy JMK, Rao CM, Meena AK. Clinical observations of juvenile myoclonic epilepsy in 131 patients: a study in South India. Seizure 1998; 7: 43-7

5. Pedersen SB, Petersen KA. Juvenile myoclonic epilepsy: clinical and EEG features. Acta Neurol Scand 1998; 97: 160-3
6. Spatt J, Langbauer G, Mamaoli B. Subjective perception of seizure precipitants: results of a questionnaire study. Seizure 1998; $7: 391-5$

7. Aziz H. Epilepsy in Pakistan: a population-based epidemiological study. Epilepsia 1994; 35: 950-8

8. Frucht MM, Quigg M, Schwaner C, Fountain NB. Distribution of Seizure Precipitants Among Epilepsy Syndromes. Epilepsia 2000; 41(12): 1534-39

9. Fountain NB, Kim JS, Lee SI. Sleep deprivation activates epileptiform discharges independent of the activating effects of sleep. J Clin Neurophysiol 1998; 15: 69-75

10. Inoue $\mathrm{Y}$, Kubota $\mathrm{H}$. Juvenile myoclonic epilepsy with praxisinduced seizures. In: Schmitz B, Sander T, editors. Juvenile myoclonic epilepsy: the Janz syndrome. Petersfield: Wrightson; 2000. p.73-81.

11. Nakken KO, Solaas MH, Kjeldsen MJ, Friis ML, Pellock JM, Corey LA. Which seizure-precipitating factors do patients with epilepsy most frequently report? Epilepsy and behavior 2005; 5: 85-89

12. Ishida S. Photosensitive seizures provoked while viewing "Pocket Monsters," a made-for-television program in Japan. Epilepsia 1998; 93: 1340-4

13. Heckmatt J, Shaikh A, Swash M, Scott D. Seizure induction by alcohol in patients with epilepsy: experience in two hospital clinics. J R Soc Med 1990; 83 